\title{
Subcutaneous peginterferon $\beta$-1a injection-site reaction experience and mitigation: Delphi analysis of the ALLOW study
}

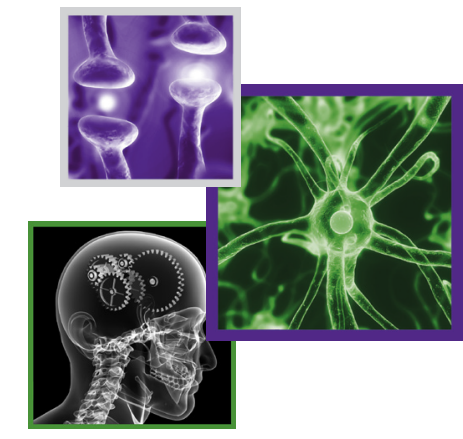

Barry Hendin ${ }^{*, 1}$, DeRen Huang ${ }^{2}$, Sibyl Wray ${ }^{3}$, Robert T Naismith ${ }^{4}$, Sheri Rosenblatt ${ }^{5}$, Javier Zambrano ${ }^{6}$ \& Brian Werneburg ${ }^{6}$

\section{Summary Points}

- Healthcare professionals agreed that erythema is the most common injection-site reaction (ISR) associated with peginterferon $\beta$-1a treatment.

- The healthcare professionals felt that the erythema was not disruptive to the patients' daily activities.

- Education provided by healthcare professionals is vital for managing patients' expectations about the potential for developing ISRs, alleviating anxiety when these adverse events occur and providing guidance on management strategies.

- It was agreed that an on-site conversation with a clinician was considered to be the best way to deliver education.

- Survey respondents mostly agreed that medication for mitigation of ISRs was not needed.

- Informing patients about the timing, impact, and management of ISRs may help to optimize treatment adherence and clinical outcomes with peginterferon $\beta$-1a.

Aim: The objective of this Delphi analysis was to obtain consensus on injection-site reaction (ISR) experience and mitigation strategies for patients with relapsing-remitting multiple sclerosis switching from nonpegylated interferons (IFNs) to peginterferon $\beta-1 a$ in the ALLOW Phase IIIb trial using a three-step approach. Methods: Study investigators and coordinators from investigative sites enrolling four or more patients in ALLOW participated in three rounds of questionnaires and interviews. Results: Respondents $(n=37)$ agreed that the most common ISR, erythema, was not disruptive to daily activities. Patient education, as a conversation with a clinician about ISR potential, was recommended. Conclusion: The consensus of Delphi respondents on ISR experience and ISR management after switching from nonpegylated IFNs to peginterferon $\beta$-1a can help inform treatment decisions and manage patient expectations.

First draft submitted: 19 July 2016; Accepted for publication: 21 October 2016; Published online: 10 January 2017

Patient adherence to disease-modifying treatments (DMTs) in multiple sclerosis (MS) is important for optimizing clinical outcomes and minimizing healthcare burden [1-4]. However, factors associated with parenteral administration of DMTs, such as frequency of administration and adverse

'Phoenix Neurological Associates, Phoenix, AZ, USA

${ }^{2}$ Neurology \& Neuroscience Associates, Akron, OH, USA

${ }^{3}$ Hope Neurology, Knoxville, TN, USA

${ }^{4}$ Department of Neurology, Washington University in St Louis, St Louis, MO, USA

${ }^{5}$ fit2market, New York, NY, USA

${ }^{6}$ Biogen, Cambridge, MA, USA

*Author for correspondence: BHendin@pnal.net

\section{KEYWORDS}

- adverse event

management • Delphi

- peginterferon $\beta-1 a$

Future Medicine part of 
events (AEs), are major barriers to adherence in patients with MS [5-7]. Large observational studies have shown that DMTs with more frequent administration are associated with lower adherence than those administered less frequently: adherence rates of 49-79\% were reported with once-daily subcutaneous (sc.) glatiramer acetate versus $77-94 \%$ for once-weekly intramuscular IFN $\beta$-1a [7-11]. Injection-site reactions (ISRs) are among the most common AEs leading to switching from or discontinuing a DMT [5,12], and they can occur frequently [13]. In a recent open-label, prospective observational study, more than 55\% of patients receiving sc. interferons or glatiramer acetate experienced ISRs [13].

Peginterferon $\beta$-1a (pegylated IFN $\beta$-1a), administered sc. every 2 weeks is approved for the treatment of relapsing MS (RMS, in the USA) and relapsing-remitting MS (RRMS, in the EU), based on results from the ADVANCE Phase III pivotal study [14]. Peginterferon $\beta$-1a demonstrated significant improvements in several clinical and MRI efficacy end points compared with placebo, and a safety profile consistent with other $\beta$ IFNs, with a lower dosing frequency than other available DMTs [14-16]. The most common AEs were flu-like symptoms (FLS) and ISRs, and erythema was the most frequently reported ISR, occurring in $62 \%$ of patients at 48 weeks [15] and $64 \%$ of patients at 2 years [16] during treatment with sc. peginterferon $\beta$-1a every 2 weeks.

Understanding the impact and management of ISRs associated with peginterferon $\beta$-1a therapy may help to improve patient adherence and clinical outcomes $[1,2,6,7,17]$. The Delphi technique, which is a well established methodology for building consensus through iterative rounds of questionnaires [18], was utilized to better characterize ISRs reported during the ADVANCE study and to identify management strategies for these AEs [19]. ADVANCE study investigators with a predefined number of enrolled patients participated in a consensus generating exercise using a modified, two-round, sequential Delphi technique. Overseen by an independent steering committee of expert clinicians, questionnaires were developed to evaluate the frequency, duration, impact and management of ISRs (questionnaire one) and then, based on the responses to the first questionnaire, to gain consensus on the impact and management of these AEs (questionnaire two). Responders agreed that ISR frequency reduced with time, erythema was the most common ISR, and the impact of ISRs on activities of daily living was minimal. Nonpharmacological management strategies were recommended for ISRs, including rotation of injection site, patient education, administering peginterferon $\beta$-1a at room temperature and cooling the injection site after injection.

Specific ISR characteristics were not obtained in the peginterferon $\beta$-1a ADVANCE study. Although the ADVANCE Delphi analysis provides important information about peginterferon $\beta$-1a related ISRs, this study was unable to obtain consensus on ISR onset or duration $[15,19]$. Additionally, the ADVANCE patient population was almost completely IFN naive; therefore, the impact of ISRs in patients switching from nonpegylated IFN therapies to peginterferon $\beta$-1a has not been investigated. The Phase IIIb ALLOW study (NCT01939002) is an ongoing, multicenter, open-label, randomized study being conducted in the USA to characterize FLS and other AEs in RMS patients who switch from nonpegylated interferon- $\beta$ therapies to sc. peginterferon $\beta$-1a. The objective of the analysis reported in this study was to gain further understanding from clinical trial researchers of the experience and impact of peginterferon $\beta$-1a-related ISRs and to obtain consensus on the most effective mitigation strategies for ISRs in relapsing-remitting MS patients switching from nonpegylated IFN therapies by applying the Delphi method to the ALLOW study using a three-step process.

\section{Methods}

In this study we performed a three-step decisionmaking process to build consensus, from study personnel taking part in the ALLOW study, on ISR experience and impact, to identify potential strategies to manage the most common ISRs and to rank patient education delivery methods. To obtain consensus, we combined quantitative and qualitative methods (online survey, qualitative telephone interviews).

\section{- ALLOW study}

The ALLOW study included patients aged 18-65 years with a confirmed diagnosis of RMS (defined by McDonald criteria [20,21]), an Expanded Disability Status Scale score between 0.0 and 5.0, and who had been receiving a stable dose of IFN- $\beta$ therapy for $\geq 4$ months. After a 4 -week run-in period during which patients remained on their nonpegylated IFN therapy, 
patients were switched to sc. peginterferon $\beta$-1a administered every 2 weeks, randomized to either their current FLS treatment or naproxen. The peginterferon $\beta$-1a dose was titrated from $63 \mathrm{mcg}$ at baseline to $125 \mathrm{mcg}$ at week 4 and this dose was maintained to week 48 . The primary objective of the ALLOW study was to determine the proportion of patients who experience new and/or increased FLS after switching from nonpegylated IFN- $\beta$ therapies to sc. peginterferon $\beta-1$. Secondary objectives included evaluating the incidence and frequency of other AEs throughout the study period. The ALLOW study was conducted in accordance with the Declaration of Helsinki, the International Conference on Harmonization and Good Clinical Practice guidelines and local regulatory requirements.

Of 201 patients randomized, 164 (81.6\% within each arm) completed the ALLOW study.

\section{- Delphi study participants}

Investigative sites with at least four patients enrolled in the ALLOW clinical trial were identified by the study medical team and invited by email to participate in the Delphi study. The sites handled by the principal investigators (PIs) in the steering committee were excluded from the study. The only information collected about participants was name, role (PIs or study coordinators [SCO]) and contact information.

\section{- Material \& data collection}

Three sequential rounds of questionnaires and qualitative interviews (only round 3 generated quantitative data) were administered to PIs and SCOs. Development of the surveys and qualitative interview questions were overseen by an independent steering committee of four neurologists, who are also authors of this manuscript (B Hendin, DR Huang, S Wray and RT Naismith). At each stage, the steering committee convened to review the data to see if consensus had been achieved and recommend next steps.

In an effort to achieve consensus, each of the three rounds of the study built on the insights obtained in the previous round. In theory, consensus could be achieved in any round. Round 1 was an online survey (Supplementary Figure 1) built in Survey Monkey, designed to obtain opinion on ISR experience and impact, as well as an initial assessment of potential strategies for resolving the most common ISRs. Open-ended questions were used throughout as a vehicle to encourage unguided ideas. A pilot of this survey was conducted with one PI and one SCO. The first round yielded the following qualitative issues for round 2: understand what is driving attitudes toward ISRs and understand perceptions of suggested approaches.

Round 2 comprised qualitative telephone interviews with round 1 responders and nonresponders to gain further insight into ISR experience and to obtain consensus on the most effective mitigation strategies for peginterferon $\beta$-1a-related ISRs. The discussion guide for round 2 has been provided as Supplementary Figure 2 . Since the steering committee decided that consensus had not been achieved, it recommend an online survey similar to round 1 as the next step, and recommended additional topics, including detailed definition of patient education, and explanation of injection-site rotation.

Round 3 was an online survey designed to assess ISR experience and provide quantified validation of the recommended mitigation strategies for peginterferon $\beta$-1a-related ISRs. The online survey for round 3 has been provided as Supplementary Figure 3.

\section{- Data analysis}

Delphi analysis results were derived from participants' responses to the round 3 survey statements about ISR experience and mitigation strategies. Statements were rated by reviewers and the means of all ratings were determined. Most statements were rated on a 1-10 rating scale, because it is more sensitive at measuring

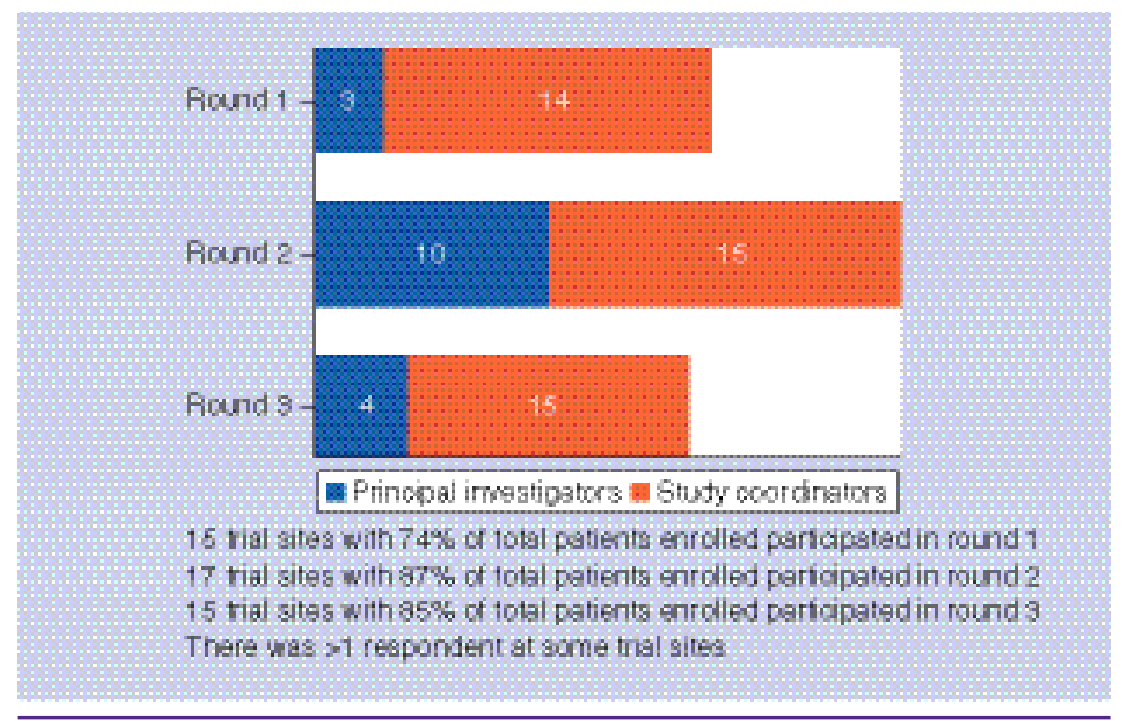

Figure 1. Respondents and trial site participation in each round of the ALLOW Delphi study. 
differences (smaller sample size required for the same degree of precision), has greater statistical reliability and validity, has been used in previous Delphi analyses, and is preferred by respondents compared with a scale with fewer points [22]. An online self-administered questionnaire allows use of rating scales that can be analyzed with standard metrics - mean and percentages which in turn can be turned into tables and graphs. Qualitative results require more subjective interpretation, typically supported by respondent quotes. The use of both analytic approaches combines objective measures with the richness of deeper insight.

\section{Results}

- Study sites \& participants

All of the ALLOW trial sites $(n=17)$ with at least four patients enrolled participated in at least one round of the ALLOW Delphi study (Figure 1); in total, 37 responded (13 PIs, 24 SCOs). The trial sites responding accounted for greater than $80 \%$ of the 201 patients enrolled in the 48 week ALLOW trial: patient mean age was 50 years; gender was $81 \%$ female; race was 90\% Caucasian and 8\% African-American; mean MS duration was 13.5 years. At the time when the surveys and interviews were carried out, the study data on ISR frequency at their site or throughout the study were not available to any of the researcher participants.

\section{- Consensus achieved}

The results following the three-step process are presented below. Consensus was achieved following the third round of data collection.

\section{- ISR experience}

For patients starting on peginterferon $\beta$-1a, the researcher respondents agreed that erythema was the most common ISR (mean agreement: $8.95 / 10$ ) but was not disruptive to the patients' daily activities (8.58/10; Figure 2). Onset of erythema was often delayed following injection, typically by more than $24 \mathrm{~h}(9.00 / 10$; Figure 2$)$, with $89.4 \%$ of responders indicating an onset after 1-4 days (Table 1). However, there were lower levels of agreement that the delayed onset of erythema was unexpected (6.32/10; Figure 2). Respondents indicated that erythema lasted longer than expected, usually $>3$ days $(8.63 / 10$; Figure 2), with the majority of PIs reporting that the median duration of erythema was 7-9 days, and the majority of SCOs reporting that the median duration of erythema was 12-14 days (Figure 3), and that duration of erythema was

Statement

$$
1=\text { Do not agree }
$$

$10=$ Strongly agree

For most patients starting on peginterferon $\beta-1 \mathrm{a}$, the most common ISR was erythema

While erythema was common, it was usually not disruptive to the patient's daily activities

The onset of erythema was often delayed after injection, typically for more than $24 \mathrm{~h}$

For many patients, the erythema lasted longer than expected, usually more than 3 days

The duration of erythema was longer than most patients had experienced with ISRs from previous interferon therapies

Other injection site reactions reported are pruritus, pain, edema, and bruising. These ISRs tend to occur much less frequently than erythema

For many patients, the delayed onset of erythema was unexpected

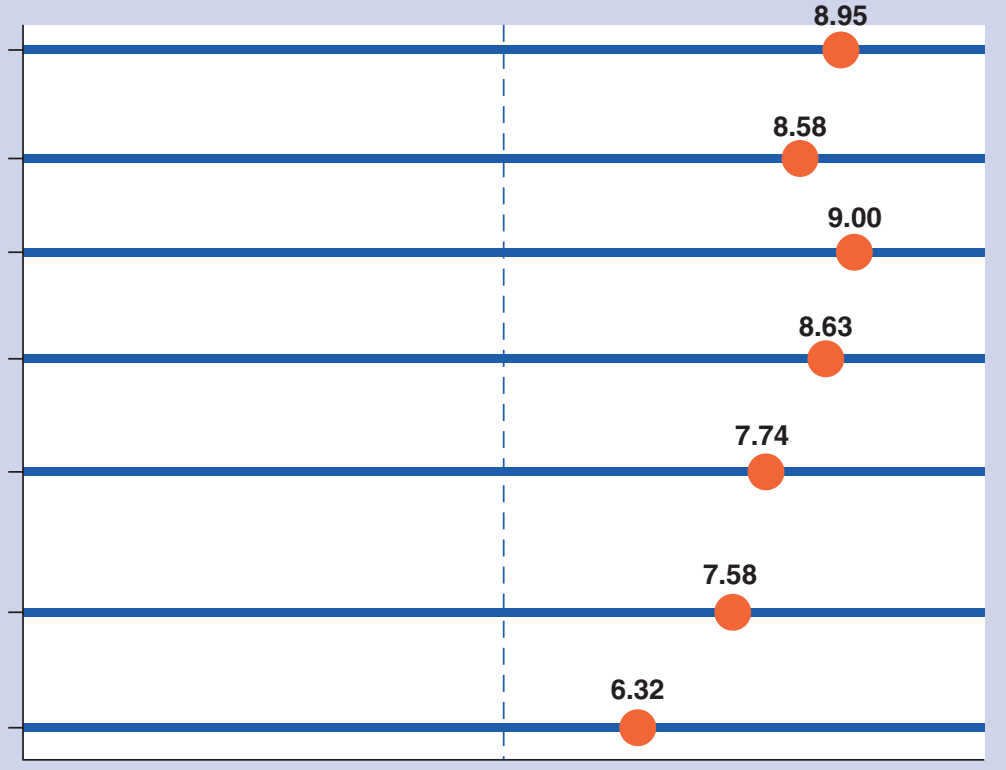

Figure 2. Consensus on injection-site reaction experience in patients switching to subcutaneous peginterferon $\beta$-1a. Data are presented as the means of all responder ratings. Consensus was achieved following round 3.

ISR: Injection-site reaction. 
Table 1. Typical onset of erythema after switching to subcutaneous peginterferon $\beta-1 a$.

\begin{tabular}{|llll|}
\hline \multirow{2}{*}{ Erythema onset } & \multicolumn{3}{c|}{ Proportion of respondents reporting typical delay $(\%)$} \\
\cline { 2 - 4 } & Study co-ordinators $(\boldsymbol{n}=15)$ & Primary investigators $(\boldsymbol{n}=4)$ & Overall $(\boldsymbol{n}=19)$ \\
\hline $1-2$ days & 6.7 & 0 & 5.3 \\
\hline $3-4$ days & 26.7 & 75.0 & 36.8 \\
\hline $5-6$ days & 60.0 & 25.0 & 52.6 \\
\hline 7 days or more & 0.7 & 0 & 5.3 \\
\hline Total responses & 100 & 0 & 0 \\
\hline Consensus was achieved following round 3. & 100 & 100 \\
\hline
\end{tabular}

longer than patients experienced with other IFN therapies (7.74/10). SCOs and PIs appeared to be in agreement about erythema prevalence, impact, onset and duration, although fewer PIs participated in round $3(n=4)$.

\section{- ISR mitigation strategies}

As a general strategy, patient education about the potential for developing ISRs was agreed to be effective for diminishing patient anxiety when ISRs did occur (mean agreement: 9.11/10; Figure 4). High levels of agreement were also found for the following preinjection statements: reassuring patients that redness is common, often delayed, and usually does not disrupt daily activities, is important (9.63/10); illustrations of a range of erythema/redness will help set expectations $(8.68 / 10)$; and using injection sites that are less visible can address the cosmetic issue of redness (8.16/10; Figure 4). Regarding postinjection statements, consensus was reached that while injection site rotation is advisable, redness may persist for many patients $(9.00 / 10)$ and patient follow-up after the first injection will help ensure that ISRs are not causing more concern than expected (8.42/10; Figure 4). Agreement regarding postinjection intervention strategies was varied, with consensus that medication is not required for most patients if expectations for erythema/redness are managed in advance $(9.16 / 10)$, although nonsteroidal anti-inflammatory drugs can be taken as needed for pain $(8.16 / 10)$. Less agreement was met regarding whether diphenhydramine or hydrocortisone cream can be helpful for relieving pruritus (6.00/10; Figure 4).

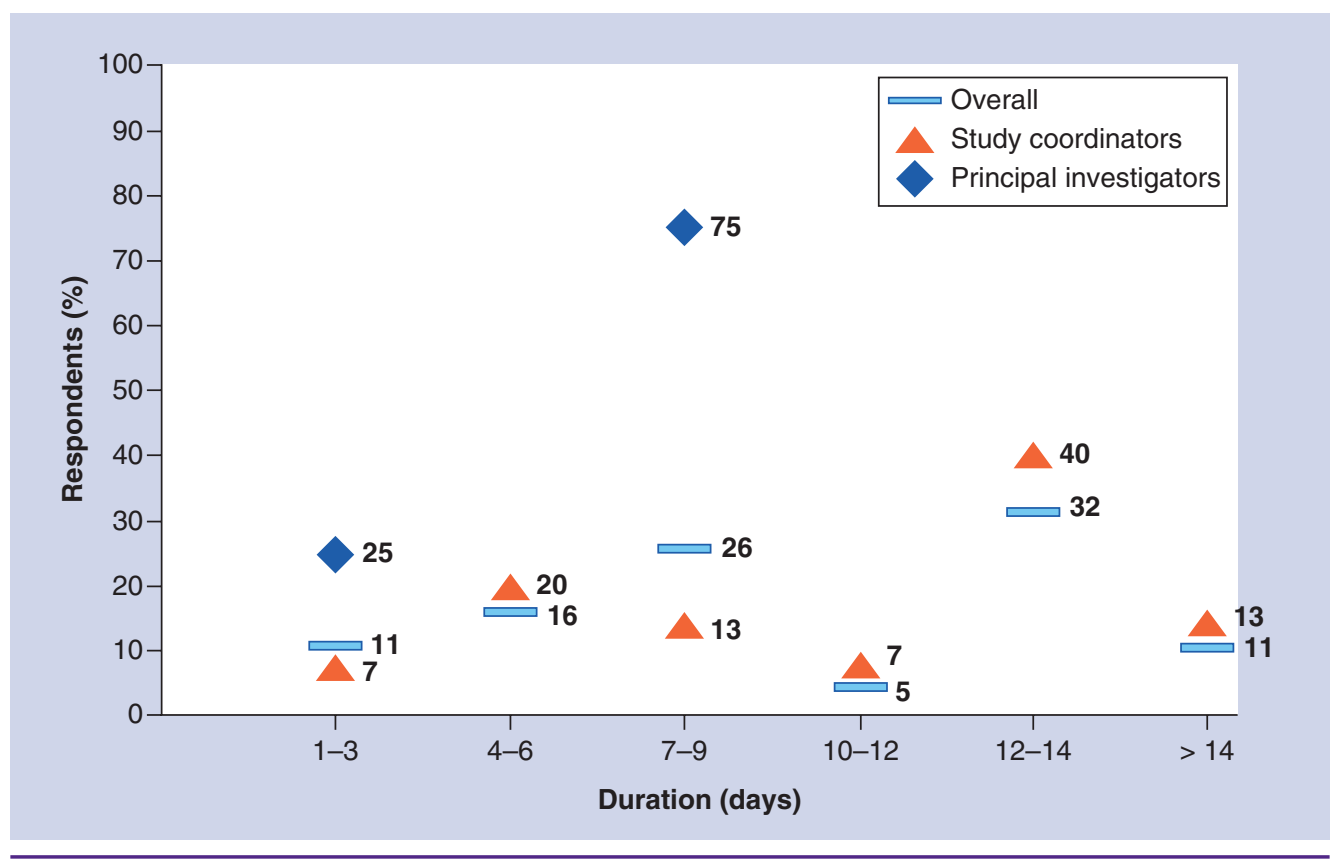

Figure 3. Typical duration of erythema after switching to subcutaneous peginterferon $\beta$-1a. Consensus was achieved following round 3. 


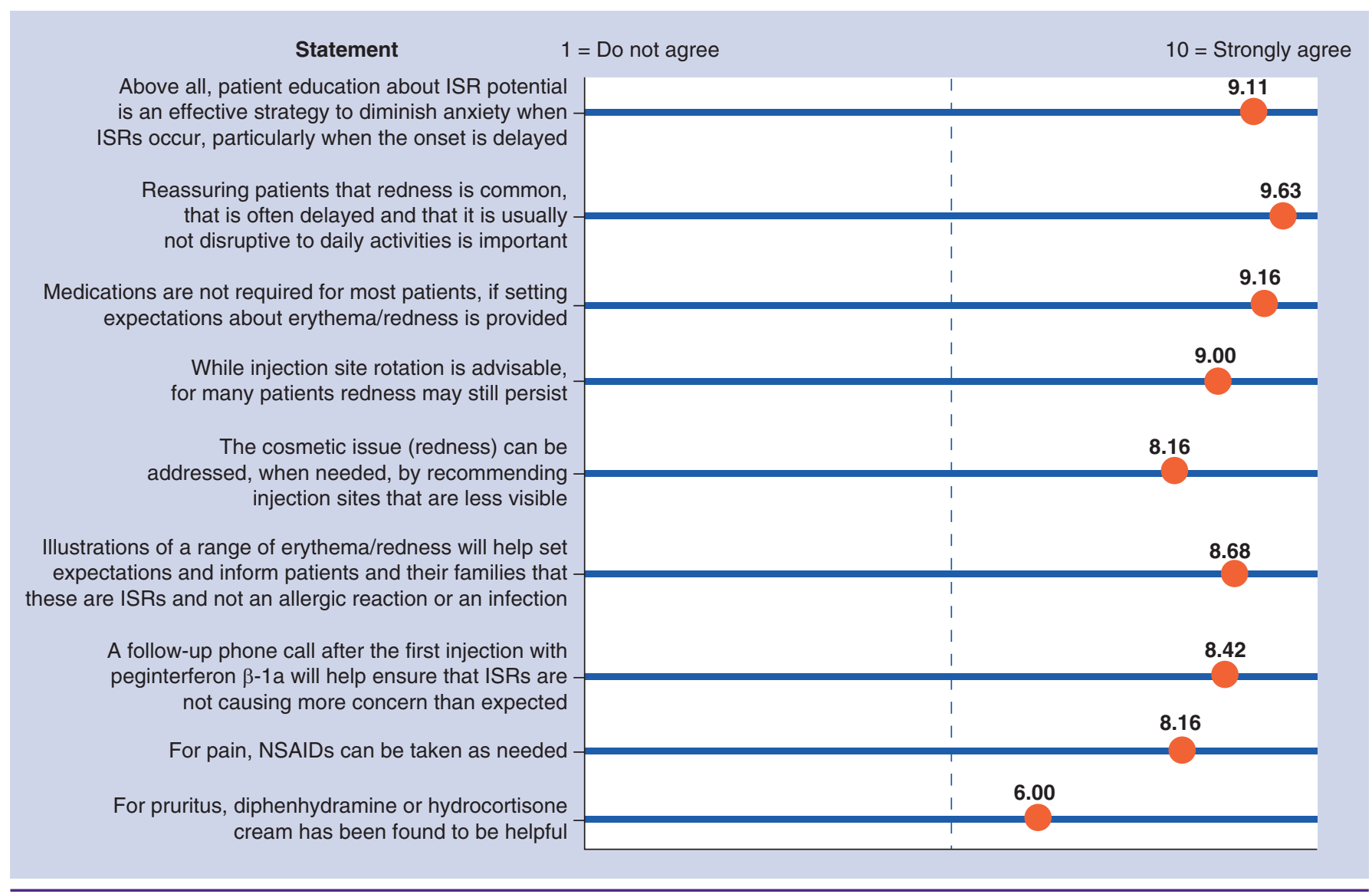

Figure 4. Consensus on mitigation strategies for injection-site reactions occurring after switching from nonpegylated IFNs to subcutaneous peginterferon $\beta-1$ a. Data are presented as the means of all responder ratings. Consensus was achieved following round 3.

ISR: Injection-site reaction.

Clinician and nurse engagement were ranked highest for delivering patient education (Table 2). An on-site conversation with a clinician was considered to be the best way to deliver education by the highest percentage of responders (42.1\%), followed by review of a brochure (containing images of ISRs) with injection training delivered by a nurse (31.6\%).

Data for discontinuations due to ISRs or FLS and a correlation of this with mitigation strategies used, are not available for the subset of study sites participating in this analysis. In the overall study, $6.0 \%$ of patients discontinued treatment due to ISRs and $2.5 \%$ due to FLS.

\section{Discussion}

Applying the Delphi technique in the ALLOW clinical trial enabled consensus to be obtained about the experience and management of ISRs associated with peginterferon $\beta$-1a treatment from the point of view of study researchers.
ISRs are common AEs with injectable DMTs and are a primary reason for treatment nonadherence and discontinuation [5,12,23-24]. In ADVANCE, the most common ISR for patients receiving peginterferon $\beta$-1a every 2 weeks in year 1 was erythema $(62 \%)$, followed by pain (15\%) and pruritis (13\%) [15]. The present analysis demonstrated agreement among Delphi responders that erythema is the most common ISR after treatment with peginterferon $\beta-1 \mathrm{a}$, but that its impact is minimal, rarely disrupting patients' daily activities or requiring medication. Responders also agreed that onset of erythema is often delayed by at least a day and tends to last longer than patients expect. Lower consensus was reached for whether erythema lasted longer than reported with other IFN therapies or whether delayed onset was unexpected.

Consistent with a wide body of previous research, consensus was reached among 
Delphi responders that patient education is the most effective mitigation strategy for ISRs. Therefore, before initiating treatment, patients should be informed regarding the potential occurrence of ISRs to help to set expectations and alleviate anxiety, particularly since the appearance of these adverse effects may often be delayed $[25,26]$. One study on IFN therapy in patients with RMS reported that MS patients often expect to experience high levels of anxiety before injections [27]. In a second study investigating patient expectations and experiences of sc. IFN therapy, potential side effects were identified as one of the most common concerns [28]. Ensuring that patients are well informed before initiating treatment improves their treatment experience and helps to prevent or delay treatment discontinuation [28]. The best way to deliver patient education was considered to be via an on-site conversation with a healthcare professional, primarily a clinician. Such proactive involvement of clinicians in patient education about MS treatment is likely to help maximize adherence and optimize patient outcomes [8]. Education delivered by nurses was also ranked highly, which supports previous research demonstrating the benefits of proactive nurse contact to promote adherence during treatment with DMTs [29]. Optimizing the quality of interaction with the healthcare team will help patients feel comfortable communicating their worries about treatment, providing the opportunity to address concerns and enhance adherence [25].

The consensus reached about the minimal impact of ISRs and recommendations of the use of nonpharmacological management strategies in the ALLOW Delphi study is consistent with the findings from the Delphi analysis in the ADVANCE study [19]. Moreover, the consensus that erythema is the most common ISR is in agreement with the AE profile for peginterferon $\beta-1 \mathrm{a}$ in the ADVANCE clinical trial $[15,16]$ and also in an interim analysis of the first 12 weeks of the ALLOW clinical trial, which reported that $29 \%$ of patients developed injection-site erythema after switching from nonpegylated IFNs, with no cases of erythema or other ISRs reported as severe [30]. As ISRs are related to administration frequency, patients would be expected to experience a lower frequency of ISRs with peginterferon $\beta$-1a than other available DMTs, which are dosed 2-14-times more often [14]. Reduced dosing frequency may help to explain why peginterferon $\beta$-1a-related ISRs were considered to have minimal impact by the ALLOW and ADVANCE Delphi responders [19] and low ISR-related discontinuation rates have been reported in clinical trials [15-16,30]. The consensus that peginterferon $\beta$-1a-related erythema has a delayed onset and tends to last longer than expected is a novel finding of the present study, as detailed characteristics of ISRs have not been reported in clinical trials, and the ADVANCE Delphi study did not reach consensus on ISR onset or duration [19].

The main limitations of this study are that the number of participants was limited to the trial sites with at least four patients enrolled in ALLOW and that data collected in the first and third round of the study were each based on surveys reliant on participant recollection. However, the three-stage sequential design of the study, the qualitative interview in the second round and the high degree of consensus obtained in the third round support the validity of our results. Another limitation of the study is the variation in participation through each round and the potential for different individuals at a given site to be completing different rounds. Additionally, as always, limited conclusion can be drawn from studies employing self-reported surveys and phone interviews. Further experience with peginterferon $\beta$-1a in real world clinical practice is needed to confirm these findings.

Table 2. Agreement on the best way to deliver patient education about injection-site reactions by study coordinators' and primary investigators' responses combined.

Patient education method

Proportion of respondents giving ranking $(n=19) ;(\%)^{\dagger}$

\begin{tabular}{|c|c|c|c|c|c|}
\hline & 1 & 2 & 3 & 4 & Total \\
\hline On-site conversation with clinician & 42.1 & 15.8 & 15.8 & 26.3 & 100 \\
\hline Brochure reviewed with nurse injection training ${ }^{\ddagger}$ & 31.6 & 26.3 & 21.1 & 21.1 & 100 \\
\hline On-site conversation and brochure & 15.8 & 52.6 & 26.3 & 5.3 & 100 \\
\hline Brochure to use on site in setting expectations about ISRs & 10.5 & 5.3 & 36.8 & 47.4 & 100 \\
\hline
\end{tabular}




\section{Conclusion}

Confirming and extending the pivotal Phase III clinical trial results, the ALLOW Delphi consensus data indicate that erythema is the most commonly observed ISR associated with peginterferon $\beta$-1a, but its impact on daily activities is minimal, as judged by clinical trial researchers. These data also highlight that educating patients on the characteristics and management of ISRs before starting treatment is considered important for setting treatment expectations, promoting adherence and improving patient outcomes.

This study is the first to provide consensus on ISR experience and mitigation strategies in patients switching from nonpegylated IFN therapies to peginterferon $\beta$-1a. The agreement obtained on ISR management is consistent with previously published recommendations for proactive patient education delivered by healthcare professionals and has the potential to impact clinical practice by ensuring optimal adherence to injections and improving therapeutic outcomes.

\section{Supplementary data}

To view the supplementary data that accompany this paper please visit the journal website at: http://www.futuremedicine.com/doi/full/10.2217/nmt-2016-0032

\section{Acknowledgements}

The authors would like to thank the study site personnel who participated in the ALLOW Delphi study.

\section{Author contributions}

All authors were involved in reviewing the manuscript critically for important intellectual content. The authors had full editorial control of the manuscript and provided their final approval of all content.
Financial \& competing interests disclosure

The ALLOW Delphi study was funded by Biogen (Cambridge, MA, USA). B Hendin is a paid consultant and/or speaker for Acorda, Biogen, EMD Serono, Genzyme, Mallinckrodt, Novartis and Teva. D Huang is a paid consultant and/or speaker for Biogen, Novartis and Teva Neuroscience. S Wray is a paid consultant, speaker andlor contract researcher for Acorda, Bayer, Biogen, EMD Serono, Genzyme, Novartis, Questcor, Receptos, Genentech/Roche and Teva. RT Naismith is a consultant andlor speaker for Acorda, Alkermes, Biogen, EMD Serono, Genentech, Genzyme, EMD Serono, Novartis, Pfizer and Questcor. S Rosenblatt has nothing to disclose. At the time of this study, J Zambrano was an employee of Biogen, and is a current stockholder of Biogen. B Werneburg is an employee and stock holder of Biogen. The authors have no other relevant affiliations or financial involvement with any organization or entity with a financial interest in or financial conflict with the subject matter or materials discussed in the manuscript apart from those disclosed.

Writing and editorial support was provided by $T$ Williamson, a professional medical writer contracted to CircleScience (Tytherington, UK). Funding for writing and editorial support was provided by Biogen (Cambridge, MA, USA).

\section{Ethical conduct of research}

The authors state that they have obtained appropriate institutional review board approval or have followed the principles outlined in the Declaration of Helsinki for all human or animal experimental investigations. In addition, for investigations involving human subjects, informed consent has been obtained from the participants involved.

\section{Open access}

This work is licensed under the AttributionNonCommercial-NoDerivatives 4.0 Unported License. To view a copy of this license, visit http://creativecommons.org/ licenses/by-nc-nd/4.0/

\section{References}

Papers of special note have been highlighted as:

- of interest; $\bullet$ of considerable interest

1 Steinberg SC, Faris RJ, Chang CF, Chan A, Tankersley MA. Impact of adherence to interferons in the treatment of multiple sclerosis: a non-experimental, retrospective, cohort study. Clin. Drug Investig. 30 (2), 89-100 (2010).

2 Cohen BA, Coyle PK, Leist T, Oleen-Burkey MA, Schwartz M, Zwibel H. Therapy optimization in multiple sclerosis: a cohort study of therapy adherence and risk of relapse. Mult. Scler. Relat. Disord. 4(1), 75-82 (2015).
3 Yermakov S, Davis M, Calnan M et al. Impact of increasing adherence to disease-modifying therapies on healthcare resource utilization and direct medical and indirect work loss costs for patients with multiple sclerosis. $J$. Med. Econ. 18(9), 711-720 (2015).

4 Lizan L, Comellas M, Paz S, Poveda JL, Meletiche DM, Polanco C. Treatment adherence and other patient-reported outcomes as cost determinants in multiple sclerosis: a review of the literature. Patient Prefer. Adherence 8, 1653-1664 (2014).

5 Costello K, Kennedy P, Scanzillo J. Recognizing nonadherence in patients with multiple sclerosis and maintaining treatment adherence in the long term. Medscape J. Med. 10(9), 225 (2008).

6 Treadaway K, Cutter G, Salter A et al. Factors that influence adherence with diseasemodifying therapy in MS. J. Neurol. 256(4), 568-576 (2009).

7 Devonshire V, Lapierre Y, Macdonell R et al. The Global Adherence Project (GAP): a multicenter observational study on adherence to disease-modifying therapies in patients with relapsing-remitting multiple sclerosis. Eur. J. Neurol. 18(1), 69-77 (2011). 
8 Remington G, Rodriguez Y, Logan D, Williamson C, Treadaway K. Facilitating medication adherence in patients with multiple sclerosis. Int. J. MS Care 15(1), 36-45 (2013).

9 Reynolds MW, Stephen R, Seaman C, Rajagopalan K. Persistence and adherence to disease modifying drugs among patients with multiple sclerosis. Curr. Med. Res. Opin. 26(3), 663-674 (2010).

10 Biogen, prescribing information. Avonex. www.avonex.com/content/dam/commercial

11 Teva-Neuroscience, prescribing information. Copaxone.

www.accessdata.fda.gov/drugsatfda_docs

12 Beer K, Muller M, Hew-Winzeler AM et al. The prevalence of injection-site reactions with disease-modifying therapies and their effect on adherence in patients with multiple sclerosis: an observational study. BMC Neurol. 11, 144 (2011).

13 Marziniak M TK, Rehberg-Weber K, Wernsdörfer C, for the Asset Study Group. AVONEX ${ }^{\circledast}$ Assessment in Multiple Sclerosis-Therapy (ASSET) - an observational study in 1343 patients. ENS 2013. www.vfa.de/nisDocument/488

14 Tolley K, Hutchinson M, You X et al. A Network meta-analysis of efficacy and evaluation of safety of subcutaneous pegylated interferon beta-la versus Other injectable therapies for the treatment of relapsing-remitting multiple sclerosis. PLoS ONE 10(6), e0127960 (2015).

- Safety of peginterferon $\beta$-1a versus other therapies.

15 Calabresi PA, Kieseier BC, Arnold DL et al. Pegylated interferon beta-1a for relapsingremitting multiple sclerosis (ADVANCE): a randomised, Phase III, double-blind study. Lancet Neurol. 13(7), 657-665 (2014).

- Year 1 of the ADVANCE pivotal peginterferon $\beta$-1a trial.

16 Kieseier BC, Arnold DL, Balcer LJ et al. Peginterferon beta-1a in multiple sclerosis: 2-year results from ADVANCE. Mult. Scler. 21(8), 1025-1035 (2015).

- Year 2 of the ADVANCE pivotal peginterferon $\beta$-1a trial.

17 Poulos C, Kinter E, Yang JC, Bridges JF, Posner J, Reder AT. Patient preferences for injectable treatments for multiple sclerosis in the United States: a discrete-choice experiment. Patient 9(2), 171-180 (2016).

18 Hsu Cc SB. The Delphi technique: making sense of consensus. Pract. Assess Research Evaluation 12(10), 1-8 (2007).

-• Overview of the Delphi technique.

19 Halper J CD, Newsome Sd, Huang D, et al. Management strategies for flu-like symptoms and injection site reactions associated with peginterferon beta-1a: obtaining recommendations using the Delphi technique. Int. J. MS Care 18(4), 211-218 (2016).

- Delphi study in ADVANCE pivotal peginterferon $\beta$-1a trial.

20 Polman CH, Reingold SC, Edan G et al. Diagnostic criteria for multiple sclerosis: 2005 revisions to the "McDonald Criteria." Ann. Neurol. 58(6), 840-846 (2005).

21 Mcdonald WI, Compston A, Edan Get al. Recommended diagnostic criteria for multiple sclerosis: guidelines from the International Panel on the diagnosis of multiple sclerosis. Ann. Neurol. 50(1), 121-127 (2001).

22 Preston CC, Colman AM. Optimal number of response categories in rating scales: reliability, validity, discriminating power, and respondent preferences. Acta Psychol. (Amst.) 104(1), 1-15 (2000).

23 Stewart TM, Tran ZV. Injectable multiple sclerosis medications: a patient survey of factors associated with injection-site reactions. Int. J. MS Care 14(1), 46-53 (2012).

24 Balak DM, Hengstman GJ, Cakmak A, Thio HB. Cutaneous adverse events associated with disease-modifying treatment in multiple sclerosis: a systematic review. Mult. Scler. 18(12), 1705-1717 (2012).
25 Brandes DW, Callender T, Lathi E, O'leary S. A review of disease-modifying therapies for MS: maximizing adherence and minimizing adverse events. Curr. Med. Res. Opin. 25(1), 77-92 (2009).

26 Rio J, Porcel J, Tellez N et al. Factors related with treatment adherence to interferon beta and glatiramer acetate therapy in multiple sclerosis. Mult. Scler. 11(3), 306-309 (2005).

27 Mohr DC, Boudewyn AC, Likosky W, Levine E, Goodkin DE. Injectable medication for the treatment of multiple sclerosis: the influence of self-efficacy expectations and injection anxiety on adherence and ability to self-inject. Ann. Behav. Med. 23(2), 125-132 (2001).

28 Syed M, Rog D, Parkes L, Shepherd GL. Patient expectations and experiences of multiple sclerosis interferon beta-1a treatment: a longitudinal, observational study in routine UK clinical practice. Patient Prefer. Adher. 8, 247-255 (2014).

29 Caon C, Saunders C, Smrtka J, Baxter N, Shoemaker J. Injectable disease-modifying therapy for relapsing-remitting multiple sclerosis: a review of adherence data. J. Neurosci. Nurs. 42(Suppl. 5), S5-S9 (2010).

30 Naismith Rt HB, Wray S, You X, Sabatella G, Zambrano J. ALLOW - a Phase IIIb trial characterising flu-like symptoms in patients transitioning to pegylated interferon beta-1a: interim analysis of all patients. Presented at: ECTRIMS Congress. Barcelona, Spain, 9 October 2015 (Abstract P1114).

- Preliminary results of the ALLOW trial during which these surveys were conducted. 